\section{Ein Beitrag zur Wirkung der Meerzwiebel. Von}

Professor Th. Husemann in Gottingen.

\section{I.}

Es ist eine auffallende Thatsache, dass wir über die Wirkungsweise gerade derjenigen Medicamente, welche wir therapeutisch am häufigsten verwenden, am wenigsten aufgeklärt sind. Man braucht in dieser $\mathrm{Be}-$ ziehung nur an Chinin und Jod zu erinnern, bezüglich deren freilich die Anfänge einer gewissen Einsicht in das Wesen ihrer Action gewonnen zu sein scheinen. Indessen haben alle Forschungen über die Wirkungen des Chinins auf Oxydation und amöboide Bewegung uns keine Antwort auf die Frage geliefert, wodurch das Chinin seine wichtigste Wirkung, die Heilung der Malariakrankheiten, zu Stande bringe. Auch fïr das Jodkalium hat sich in den letzten Jahren eine Grundlage zur Erklärung der segenannten Actio resolvens des Mittels in gewissen chemischen Veränderungen, welche dasselbe im Organismus erleidet, gefunden, doch ist bis jetzt unter den Pharmakologen eine Einigung darüber nicht erzielt, ob die Abspaltung des Jods aus dem Jodkalium im Blute oder in den Geweben vor sich geht.

Zu den Substanzen, welche bei den meisten practischen Aerzten in höchsten Ansehen stehen, ohne dass ihre Wirkungsweise ihnen irgend wie genauer bekannt wäre, gehört auch die Meerzwiebel, bekanntlich eines der altesten Arzneimittel, deren Benutzung gegen Wassersucht bereits Pythagoras von den Aegyptern gelernt haben soll. Gewiss ist, dass -bei medicinischen Schriftstellern des Alterthums, Diosk orides, Celsus, Galen, die Anwendung der Scilla bei Wassersucht hervorlyeben und befürworten, woneben auch eine grössere Anzahl medicinischer Verwendungen in Krankheitszuständen sich findet, welche im Laufe der Jahrhunderte absolet geworden sind und in denen zum Theil, wie in den mit Meerzwiebel, Grünspan und anderen Ingredienzien angefertigten Pflastern gegen allerlei Geschwüre die Meerzwiebel offenbar eine ganz untergeordnete Holle spielt. Erhalten hat sich nur neben der antihydropischen Verwendung der Meerzwiebel der schon von Celsus und Plinius erwähnte Gebrauch bei Kurzathmigkeit und katarrhalischen Affectionen.

Gegenwärtig stehen bei uns noch verschiedene Präparate in Anwendung, deren die Alten sich bedienten. Pythagoras soll schon den Meerzwiebelessig bereitet haben, wie er noch heute in unseren Pharmakopöen sich findet. Eine Zubereitung mit Honig, wie sie noch heute in Frankreich als Mellitım Scillae eine Stelle in der officiellen Phar makopoe einnimmt, benutzte schon Hippokrates. Die Combination beider Präparate, den Meerzwiebelsauerhonig, finden wir seit dem ersten Jalırlıundert nach Christus erwähnt. Alle genannten Präparate müssen freilich in praxi dem Meerzwiebelextract den Vorrang lassen, der namentlich als Bestandtheil antihydropischer Pillen eine höchst ausgedelınte Auwendung findet.

Das Extractum Scillae hat in der neuen deutschen Pharmakopoe eine wesentliche Veränderung erfahren. Das Meerzwiebelextract der letzten Auflage der Pharmacopoea Borussica war ein kalt bereitetes wässriges Extract, welches ein gelbliches, in Wasser nicht ganz klar lösliches Pulver bildete. Die $\mathrm{Ph}$. Germanica lässt dagegen die grobgepulverte trockene Meerzwiebel durch 4 tägige Maceration mit 4 Theilen Spiritus dilutus (Spiritus von 0,892-0,893 spec. Gew. oder 60,75 Gewichts- oder 68,35 Volumprocent nach Angahe ler Phkp., deren alkoholometrische Angaben übrigens nicht vollkommen exact sind) ansziehen und aus dem filtrirten Auszuge ein gewöhnliches, dickliches, gelblichbraunes Extract herstellen.

Die Scilla gehört bis jetzt zu denjenigen Gewächsen, über deren wirksames Princip wir vollkommen im Unklaren sind. Was bisher über die Darstellung eines reinen Stoffes aus der Scilla publicirt worden ist, lässt uns nicht glauben, dass irgend Jemand einen solchen in Händen gehabt hat. Das Scillitin von Telloy wird als eine farblose spröde Hasse von harzahnlichem Geruch und liöchst bitterem Geschmack beschrieben, welche an der Luft Feuchtigkeit anzieht, sich in Wasser zu einer schleimigén Flüssigkeit löst und bei Anwendung von Wärme auch in absolutem Alkohol gelöst wird, während Aether und ebenso Säuren nicht darauf einwirken. Diese Substanz ist somit sicher niclit basischer Natur wie das angebliche Scillitin, welches Landerer 1834 aus frischen Meerzwiebeln dargestellt haben will. Derselbe will den Stoff in Crystallen erhalten haben, welche zwisehen den Zähnen knirschten, einen selır bitteren, dagegen nicht scharfen Geschmack besassen, leicht geröthetes Lakmuspapier bläuten, in 120 Theilen Weingeist sich lösten, dagegen in Wasser, in ätherischen und fetten Oelen und in Aether unlöslich erschienen, mit Säuren Salze bildeten und beim Erhitzen unter Ausstossung eines zu Husten reizenden Geruelss schmolzen. Das sind offenbar zwei ganz verschiedene Stoffe gewesen, die abgesehen von ihrer basischen oder nicht basischen Natur auch durch ihre Löslichkeitsverhältnisse einander schroff gegenuber stehen. Die alkaloidische Natur des Scillitins von Landerer ist schon von Bley und Wiggers in Zweifel gezogen und wenn auch Righini denselben durch die ganz unberechtigte Hypothese, dass die Meerzwiebel Veratrin enthalte, zu Hülfe gekommen ist, so ist doch keinem späteren Chemiker gelungen, obschon die Seilla wiederholt neueren Untersuchungen unterworfen ist, eine Pflanzenbase mit Sicherheit daraus zu isoliren. $\mathrm{Ob}$ es sich bei der Scilla iiberhaupt um ein einziges oder um mehrere wirksame Principien handelt, darïber liegt etwas Zuverlässiges nicht vor. Naeh Mandet (Compt. rend. LJ. 87) sollen zwei Körper sich darin finden. Der eine, das Scillitin, soll die diuretischen und expectorirenden Eigenschaften der Scilla bedingen, der andere, das Sculemn, soll die toxischen und irritirenden Wirkungen der Meerzwiebel verschulden. Es ist zu bedauern, dass ausser einer Notiz von 3-4 Reilien in den Berichten der Académie des Sciences sich nicht eine Spur über die Mandet'schen Meerzwiebelkörper in der Literatur findet; der Verfasser jener Notiz ist uns die Aufklärung darüber schuldig geblieben, wie dieselben aussehen und wie sie sich Lösungsinitteln und chemischen Reagentien gegenüber verhielten. Was im Handel unter dem Namen Scillitin existirt, ist keine als ein e anzuselıende Substanz. Eine Quantität Scillitin, welches ich durch die Giute von Merck in Darmstalt erhielt, entsprach durchaus der Angabe Merck's, dass es sich nicht um einen reinen Körper, sondern um ein gereinigtes Extract handele. Es war eine dunkelbraun gefärbte, wenig bitter schmeckende Masse, welche auch bei Vergrösserung keine Crystallisationen zeigt und wie wir weiter unten sehen werden, durch deı Reinigungsprocess des grössten Theiles des eigentlichen wirksamen Princips beraubt wurde.

Ich labe Herrn Dr. Adolf König aus Witten veranlasst, mit dem von der Pharm. Germ. eingefülirten wässrig-spirituösen Extractum Scillae einerseits und dem Scillitin von Merck andererseits verschiedene Thierversuche anzustellen, welche derselbe in seiner Inaugural-Dissertation ') ausführlicher veröffentlicht lıat. Da die Resultate dieser Versuche auf die Frage, in welcher Weise die diuretische Action der Scilla zu Stande koninit, helles Licht zu werfen sclieinen und anch andere Punkte, welche in Bezug auf die Meerzwiebel einer Aufklïrung bedürfen, ein wenig erlıellen, erlaube ich nir die Hamptsachen aus dieser Arbeit nebst einigen sich daran knüpfenden Bemerkungen in Kiirze mitzutheilen:

Als erstes Hauptresultat der in Rede stehenden Thierversuche ergiebt sich, dass es eine Unmöglichkeit ist, die toxischen und therapeutischen Wirkungen der Scilla von einander zil trennen. Wir erwähnten bereits oben, dass Mandet die diuretischen und expectorirenden Eigenschaften von einem besonderen Körper ablängig macltt, welcher verschieden von demjenigen sei, der die toxische und irritirende Action der Scilla bedingt, Es stellt sich bei unseren Versuchen heraus, dass wenigstens die diuretischen Wirkungen des Extractuin Scillae mit denjenigen Effecten in $\mathrm{Zu}$ sammenhang steheil, deren Steigerung der giftigen und deletaeren Einwirkung der Meerzwiehel auf den Organismus zu Grunde liegt. Die Scilla bietet einen Beweis dafür, wie toxikologische Studien an Thieren im Stande sind, pharmakodyılamische Streitfragen zur Lösung gelangen zu lassen und gerade in diesel Beziehung möchte die vorliegende Studie für die vereinzelten Gegner des Thierexperiments einen iiberzeugenden Beweis niclıt allein von der Zweckmässigkeit, sondern sogar von der Nothwendigkeit der Experimente lieferı.

In England hat sich neuerdings durch Harley, Kennedy u. A. die wohlbegründete Ansicht Geltung zu verschaffen gesucht, dass in sehr vielen Fällen die therapeutischen Effecte mit den plyysiologischen zusammenfallen und dass es um erstere zu haben, noch solcher Dosen bedürfe, deren Wirkung anı Organisınus durch plyysiologische Veränderungen erkannt werden können. Kennedy macht darauf aufmerksam, dass manche der älteren Arzneimittel, z. B. Conium, dadurch in Misscredit gekommen seien, dass man sie neuerdings in allzu kleiner Dosis reiche. Es ist das offenbar insoweit richtig, als es sich bei der Darreichung von Schierlingspräparaten um die Beseitigung von Nervenaffectionen handelt, während wir die von gläubigen Anhängern Störck's gerühmten Heilwirkungen des Schierlings bei bösartigen Geschwülsten weder mit physiologischen noch mit toxischen Dosen erringen können, da hier. offenbar diagnostisehe Täuscliungen mit untergelaufen sind. Da, wie wir schon gesehen haben, und weiter unten zeigen werden, auch bei der Meerzwiebel die therapeutischen und physiologischen Effecte miteinander in innigstem Zusammenlange stehen, so nag es sein, dass die Vernachlïssigung des Mittels von einigen Pharnıkologen und Aerzten sich durch die voll ilmen angewendeten allzu kleinen Gaben erklïrt. Freilich ist dies wohl nicht bei Oesterlen der Fall, welcher, wie auch bei vielen anderen Mitteln, so auch bei der Scilla die Wirksamkeit anzweifelt und bei Hydropischen in guter Kost, Wein, Pflege u. dgl. bessere Förderungsmittel die Gesundheit sicht als in der Scilla. Ein Grund zur Vernächlässigung von Seiten einzelner Kategorien von Aerzten liegt freilich darin, dass gerade die pharmakodynamischen Verhältnisse unaufgeklärt blieben und dass indem die Angaben älterer Pharmakologen sich in den Lehr- und Handbüchern der Materia medica als ewige Krankheit forterbten, gewisse Befüch-

1) Einige Untersuchungen über die Wirkungsart des Extractum Scillae Seillitin und Preverisin. Göttingen 1875. 
tuugen schädlicher Effecte der Scilla sich geltend machten und bis in die neneste Zeit hinein beunruhigend wirkou. lliese pliarmakodynamischen Voraussetzungen gipfeln darin, dass z. B. Buchheim in der orsten Auflage seines Lelırbuches die Scilla geradezı zur Gruppe des Elateriums mitten zwischen die Koloquinten und dio Gratiola postirt. Selbst nach den lö̈chst griundlichen und in ihren Resultaten mit den vorlicgenden Untersucliungen in vielen Punkten übereinstimmenten Versnchen, welche wenigstens bis zu einem gewissen Grade die Lehre von der Schärfe, die man der Seilla beilegte, berichtigten, gieltt es Lehrbüeher, wie dasjenige von Posner, in denen die Einwirkung der Scilla auf das Nervensystem, auf die durch selır grosse Gaben der Meerzwiebel hedingte lieftige Gastroenteritis und Nephritis bezogen wirl.

Eine genaue Untersuchung der älteren Literatur giebt verlıältinissmässig wenig Anlıaltspunkte für die Existenz einer wirklichelı Gastroenteritis, bei interner Application ihrer Präparate. Wir können die hautröthende Wirkung der frischen Meerzwiebel als erwiesen betrachten oder nicht; fïr die bei uns ausschliesslich zur Verwendung kommende trockne Meerzwiebel ist von Schroff der Nachweis geliefert worden, dass dieselbe eines flüchtigen Princips vollständig entbehrt.

Von einer sclarfen und brennenden Wirkung, wie sie Dioscorides der Meerzwiebel beilegt, kann bei der getrockneten Scilla keine Rede melır sein. Die Angabe von Schroff, dass höchstens die unorganischen in der frischen Meerzwiebel enthaltenen Crystalle von oxalsaurem Kalk eine gelinde Hautreizung erzeugen können, fand ihre Bestätigung. Unmittelbar durch Kralımer und mittelbar durch Flückiger (vgl. Pharmacographia p. 529), wurde gefunden, dass der schleinige Saft der nahe verwandten Agapanthus umbellatus vermöge der darin reichlich entlialtenen Crystallnadeln (Raphiden) beim Einreiben mehrstïndiges Jucken. und Röthen erzeugt. 